\title{
The clinical sensitivity of a single SARS-CoV-2 upper respiratory tract RT-PCR test for diagnosing COVID-19 using convalescent antibody as a comparator
}

\author{
Authors: Abigail Holborow, ${ }^{\mathrm{A}}$ Hibo Asad, ${ }^{\mathrm{B}}$ Lavinia Porter, ${ }^{\mathrm{C}}$ Poppy Tidswell, ${ }^{\mathrm{C}}$ Claire Johnston, ${ }^{\mathrm{D}}$ Ian Blyth, ${ }^{\mathrm{D}}$ Alice Bone ${ }^{\mathrm{E}}$ \\ and Brendan Healy ${ }^{\mathrm{F}}$
}

\begin{abstract}
The clinical false negative rate of reverse transcriptase polymerase chain reaction (RT-PCR) testing for SARS-CoV-2 on a single upper respiratory tract sample was calculated using convalescent antibody testing as a comparator. The sensitivity in symptomatic individuals was $86.2 \%(25 / 29)$. Of the missed cases, one (3.5\%) was detected by repeat RT-PCR, one by CT thorax and two (7.1\%) by convalescent antibody. The clinical false negative rate of a single RT-PCR on an upper respiratory tract sample of $14 \%$ in symptomatic patients is reassuring when compared to early reports. This report supports a strategy of combining repeat swabbing, use of acute and convalescent antibody testing and CT thorax for COVID-19 diagnosis.
\end{abstract}

KEYWORDS: COVID-19, SARS-CoV-2 RT-PCR, clinical sensitivity, COVID-19 antibody

DOI: $10.7861 /$ clinmed.2020-0555

\section{Introduction}

The clinical sensitivity of reverse transcriptase polymerase chain reaction (RT-PCR) testing for SARS-CoV-2 on a single upper respiratory tract specimen is a source of ongoing debate, partly fuelled by early reports of low sensitivity of throat swabs. 'While RT-PCR is highly specific and remains the principal method for detecting COVID-19 infection across the world, understanding the false negative rate is important so that clinicians have an estimate

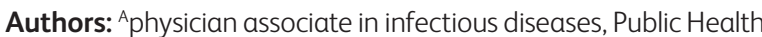
Wales Microbiology Department, Swansea, UK; Binformation analyst, Public Health Wales Microbiology Department, Swansea, UK; Cmedical student, Swansea University Medical School, Swansea, UK; ${ }^{D}$ specialist registrar in infectious diseases and microbiology, Public Health Wales Microbiology Department, Swansea, UK; E ${ }^{\text {senior house }}$ officer in infectious diseases and microbiology, Public Health Wales Microbiology Department, Swansea, UK; 'F consultant in infectious diseases and microbiology, Public Health Wales Microbiology Department, Swansea, UK of the reliability of the test when making management plans based on the results.

Determining the clinically false negative rate is difficult because until recently there was no other diagnostic test as specific as RT-PCR for determining the presence of infection. The clinical presentation is varied and so no combination of symptoms can reliably diagnose COVID-19 infection. Radiological findings on chest X-ray and CT scans can be indicative but in many instances are not sufficient to conclusively rule in or rule out COVID-19. As such, previous estimates of the sensitivity and specificity of RT-PCR were limited by the lack of a reliable specific comparator.

The specificity of antibody testing is in the region of $95-100 \%$, with sensitivity of $90-100 \% .^{2}$ Convalescent serology provides an opportunity to more precisely estimate the clinical false negative rate of a single RT-PCR test, particularly if used in combination with other tests.

In this study, we have evaluated the clinical false negative rate of a single upper respiratory tract sample in the UK by investigating two well defined clusters of infection and comparing results from in-house real-time RT-PCR testing targeting the e-gene with RNAseP used as an internal control against convalescent antibody testing, repeat RT-PCR and CT scan results (where appropriate).

\section{Methods}

Results from two clusters of infection among healthcare workers in well-defined settings were analysed using RT-PCR and convalescent antibody testing. Staff that were RT-PCR-negative were tested for antibody using EUROIMMUN Anti-SARS-CoV-2 ELISA assay for detection of IgG antibodies 6-8 weeks after the cluster of infection. The clinical false negative rate was calculated by comparing the results from a single RT-PCR swab with results from repeat swabs (where taken), CT chest (where available and strongly suggestive of COVID-19) and convalescent antibody testing. The data presented here were collected as part of routine service. The paper has been reviewed by local information governance and ethics committees and deemed suitable for publication.

\section{Results}

127 staff were working in the defined areas and had potential exposure during the outbreak period. 42 were symptomatic, 
Table 1. Number of individuals from cluster investigation broken down by symptoms, RT-PCR results and convalescent SARS-CoV-2 antibody/CT thorax findings consistent with COVID-19

\begin{tabular}{llccccc} 
& \multicolumn{3}{c}{ Symptomatic, $\mathbf{n}=\mathbf{4 2}$} & \multicolumn{3}{c}{ Asymptomatic, $\mathbf{n}=\mathbf{8 5}$} \\
& + ve & $-v e$ & NP & + ve & - ve & NP \\
First & 25 & 17 & & 10 & 63 & 12 \\
RT-PCR & & & & & & \\
Second & 1 & 5 & 11 & 1 & 3 & 59 \\
RT-PCR & & & & & & \\
Antibody & 2 & 10 & 4 & 5 & 41 & 16 \\
CT & $1^{*}$ & NA & 13 & & & \\
Total & 29 & & & 16 & & \\
\hline
\end{tabular}

* Subsequent to original submission this individual has also now tested positive for antibody. +ve = detected; - $-v e=$ not detected; $N P=$ not performed.

of whom 25 were positive following a single swab. Six of the negative-swab individuals underwent a second swab, of whom five were negative and one was positive (repeat swab 13 days after initial swab but no new symptoms in between). 13 out of 16 staff with negative RT-PCR tests underwent convalescent antibody testing and the result was positive in two and negative in 10. One of the individuals who was negative by RT-PCR but did not have convalescent antibody testing had a CT chest that was highly suggestive of COVID-19; this was considered a false negative RTPCR result for analysis. Overall, 29 symptomatic individuals were considered positive (25 first RT-PCR-positive, one second RT-PCRpositive, one CT-positive, two convalescent antibody-positive). The clinical false negative rate of a single throat swab was 14\% (4/29). There were no convalescent antibody data on three individuals.

Eighty-five individuals were asymptomatic; 73 were swabbed, 10 were positive and 63 were negative. Four were swabbed for a second time (presumably because of onset of symptoms) and one was positive (excluded from false negative analysis). Of the remaining 62 asymptomatic negative individuals, five were positive for SARS-CoV-2 IgG antibodies, 41 were negative and 17 were not tested.

Results are summarised in Table 1.

\section{Discussion}

The presentation of COVID19 is varied and non-uniform, ranging from asymptomatic infection and mild disease through to severe infection and death. ${ }^{3}$ The varied presentation and the overlap of symptoms and signs with other viral illnesses, respiratory and infection syndromes means that the clinical presentation alone cannot be relied upon for diagnosis.

None of the currently available tests are $100 \%$ sensitive or specific, resulting in an element of uncertainty when managing cases. An estimate of the clinical false negative rate is useful when managing this uncertainty given the potential implications associated with a missed diagnosis.

Concerns about the clinical sensitivity of the RT-PCR test have been fuelled by reports suggesting a high clinical false negative rate, with a sensitivity ranging from $32-70 \%$ for a single pharyngeal sample. ${ }^{1,4}$ The sensitivity of a single RT-PCR test will vary according to the disease stage (increased chance of positivity early in the illness), ${ }^{5-8}$ the site of infection (the virus may be differentially expressed at different sites during the course of the illness), ${ }^{9}$ the quality of the sampling, the analytical sensitivity of the assay and perhaps the severity of the infection. ${ }^{5,6}$

Subsequently $\mathrm{CT}$ thorax has been proposed as a diagnostic tool..$^{4,10-14} \mathrm{CT}$ scans are sensitive but lack specificity. ${ }^{15}$

Individuals will present for diagnosis at different stages of infection. Key workers seeking a diagnosis from an occupational health perspective and those tested during contact tracing will mostly present in the early phases of the illness. Those presenting to hospital can present at potentially any time point in the illness. Individuals may present in the early stages when the infection has caused some form of decompensation requiring hospital care (for example, a fall in the elderly or hyperglycaemia in diabetics). Others will present later during the respiratory phase of the illness. The sensitivity and specificity of RT-PCR, radiographic examinations and antibody tests vary according to the stage of the disease in a complementary fashion.

Antibody tests have a sensitivity and specificity in the region of $90-100 \%$. $^{2}$ The Euroimmun assay used in this study has a specificity of $95-100 \%$ and a sensitivity (which increases with time since onset of infection) of $90 \% .^{2}$ The specificity of antibody testing has been determined by detecting antibody in individuals who were positive by RT-PCR. Convalescent antibody testing facilitates retrospective diagnosis of cases that might not be detected by RT-PCR because of the timing of the sample (eg early or late on in the disease process). However, it is possible that some true cases of COVID-19 will be negative by both RT-PCR and antibody testing.

This study demonstrates that the clinical false negative rate for SARS-CoV-2 RT-PCR in symptomatic individuals in a UK setting is around $14 \%$ (Table 2 ). The strength of this study is the use of a combination of very sensitive and specific tests (antibody, repeat RT-PCR and high likelihood CT) to determine the true clinical false negative result of RT-PCR. The sensitivity and specificity of a combined diagnostic approach of RT-PCR plus acute and convalescent antibody plus CT scan or chest X-ray (when appropriate) is not known but is likely to be high. Each modality complements the other, as each has a maximum sensitivity at different stage of the disease.

Limitations of the study include the lack of acute serum and the time ( $6-8$ weeks) that elapsed from the outbreak to the convalescent antibody testing. As such, it is possible that some individuals developed infection at another time that was not related to the outbreak. This is particularly relevant in the asymptomatic group, where the high false negative RT-PCR rate may be partly explained by acquisition of infection remote from the outbreak. Others may have had mild infection and either not

Table 2. Sensitivity of single SARS-CoV-2 RT-PCR

test on healthcare staff individuals during two

clusters of infection

$\begin{array}{llll} & \text { Symptomatic } & \text { Asymptomatic } & \text { Combined } \\ \text { Cluster 1 } & 87.50 \% & 60 \% & 77 \% \\ \text { Cluster } 2 & 85 \% & 80 \% & 83 \% \\ \text { Total } & 86 \% & 67 \% & 80 \%\end{array}$


mounted a significant enough antibody response to be picked up or had a weak antibody response that had waned by the time of testing.

The sensitivity of RT-PCR of $86 \%$ is in keeping with other published studies. It is at the upper end. We believe that it is likely that some studies that have quoted a sensitivity at the lower end (eg $30 \%$ ) likely underestimate the sensitivity of RT-PCR testing in the UK through a combination of factors including incorrect diagnosis of COVID-19 in individuals with other infections and issues related to sampling and RT-PCR testing.

\section{Conclusion}

In summary we have demonstrated that in a UK centre the clinical sensitivity of a single RT-PCR on an upper respiratory tract sample is $86 \%$ when compared to repeat swab results, highly suggestive CT scan results (where available) and convalescent antibody testing in symptomatic individuals.

We suggest that patients with negative RT-PCR samples should be clinically re-evaluated and carefully assessed. Decisions in relation to clinical management and placement should be based on that assessment, taking into account the presenting symptoms and results from blood tests (including acute and convalescent antibody testing) and imaging.

Consideration should be given to re-swabbing, testing lower respiratory tract samples (where appropriate) and using acute and convalescent antibody testing and $\mathrm{CT}$ thorax and/or chest $X$-ray where appropriate to aid the diagnostic process, including retrospective diagnosis that may be useful to guide follow-up. Interpretation of all results should also take into consideration the stage of the disease based on the days that have elapsed since symptom onset.

\section{Acknowledgments}

We would like to thank Shelley Evans for her support in collating some of the data.

\section{References}

1 Wang W, Xu Y, Gao R et al. Detection of SARS-CoV-2 in different types of clinical specimens. JAMA 2020;323:1843-4.

2 US Food and Drug administration. EUA authorized serology test performance. FDA, 2020. www.fda.gov/medical-devices/ emergency-situations-medical-devices/eua-authorized-serologytest-performance.
3 Huang C, Wang Y, Li X. Clinical features of patients infected with 2019 novel coronavirus in Wuhan, China. Lancet 2020;395:497506.

4 Xie X, Zhong Z, Zhao W et al. Chest CT for typical 2019-nCoV pneumonia. Radiology 296:E41-5.

5 He X, Lau EHY, Wu P et al. Temporal dynamics in viral shedding and transmissibility of COVID-19. Nat Med 2020;26:672-5.

6 Zou L, Ruan F, Huang M et al. SARS-CoV-2 viral load in upper respiratory specimens of infected patients. N Engl J Med 2020; 382:1177-9.

7 To KK-W, Tsang OT-Y, Leung WS et al. Temporal profiles of viral load in posterior oropharyngeal saliva samples and serum antibody responses during infection by SARS-CoV-2: an observational cohort study. Lancet Infect Dis 2020;20:565-74.

8 Kluytmans M, Buiting A, Pas S et al. SARS-CoV-2 infection in 86 healthcare workers in two Dutch hospitals in March 2020. medRxiv 2020;2020.03.23.20041913.

9 Shufa Z, Jian F, Fei Y et al. Viral load dynamics and disease severity in patients infected with SARS-CoV-2 in Zhejiang province, China, January-March 2020: retrospective cohort study. BMJ 2020; 369:m1443.

10 Rubin GD, Ryerson CJ, Haramati LB et al. The role of chest imaging in patient management during the COVID-19 pandemic: a multinational consensus statement from the Fleischner Society. Chest 2020;158:106-16

$11 \mathrm{Ai}$, Yang Z, Hou H et al. Correlation of chest CT and RT-PCR testing in coronavirus disease 2019 (COVID-19) in China: a report of 1014 cases. Radiology 2020;296:E32-E40.

12 Fang $\mathrm{Y}$, Zhang $\mathrm{H}$, Xie J et al. Sensitivity of chest CT for COVID-19: comparison to RT-PCR. Radiology 2020;296:E115-7.

13 Wong HYF, Lam HYS, Fong AH-T et al. Frequency and distribution of chest radiographic findings in COVID-19 positive patients. Radiology 296:E72-8.

14 Bernheim A, Mei X, Huang M et al. Chest CT findings in coronavirus disease-19 (COVID-19): relationship to duration of infection. Radiology 2020;295:200463.

$15 \mathrm{Kim} \mathrm{H}$, Hong H, Ho Yoon S. Diagnostic performance of $\mathrm{CT}$ and reverse transcriptase-polymerase chain reaction for coronavirus disease 2019: a meta-analysis. Radiology 2020;296:E145-55.

Address for correspondence: Dr Brendan Healy, Public Health Wales Microbiology Department, 8 Eaton Cres, Sketty, Swansea SA2 8QA, UK. Email: brendan.healy@wales.nhs.uk 